\title{
On Comparison Analysis of Multiple Interference MIMO Systems with Un-Correlated Rician Fading Channels
}

\author{
Affum Emmanuel \\ Department of Electrical and \\ Electronic Engineering, \\ Kwame Nkrumah University of \\ Science and Technology, \\ Kumasi-Ghana
}

\author{
Addo Emmanuel \\ Department of Electrical and \\ Electronic Engineering, \\ Kwame Nkrumah University of \\ Science and Technology, \\ Kumasi-Ghana
}

\begin{abstract}
This paper evaluates the multiple-input multiple-output (MIMO) wireless systems in Uncorrelated Racian interference Channel using the successive decoding approach. The analytical and simulation result indicates that the MIMO system provides promising estimates for average BER performance. The extension of MIMO system with $n_{R}$ receive antennas and $n_{T}$ transmit antennas to multiple interfering transmitter under the assumption of a common receive correlation matrix indicates that the Racian factor and SNR depend on the full transmit covariance matrices. Also, the numerical result obtained favors large number of Antennas in applicative point of view as the performance of system with larger number of Antennas seems less affected by the variance and the multiple interferes
\end{abstract}

\section{General Terms}

Racian Factor, Nuttall $Q$-function, Covariance matrices

\section{Keywords}

MIMO, Uncorrelated, Multiple Interference

\section{INTRODUCTION}

Vector channels, or multiple-input multiple-output (MIMO) channels, represent a very general description for a wide range of applications. They incorporate SISO (Single-Input SingleOutput), MISO (Multiple-Input Single-Output) and SIMO (Single-Input Multiple-Output) channels as special cases. Often, MIMO channels are only associated with multiple antenna systems. However, they are not restricted to this case but can be used in a much broader context, for example, for any kind of multiuser communication. Principally, single-user and multiuser communications are distinguished. In the single user case, the multiple inputs and outputs of a vector channel may correspond to different transmit and receive antennas, carrier frequencies, or time slots due to the fact that the data stems from a single user
[1]. By transmitting parallel data streams over a MIMO channel, it was shown that the Shannon capacity of MIMO channel increases linearly with the minimum number of transmits and receives antennas in [2] and [3]. Compared with single input single-output (SISO) systems, MIMO system leads to a dramatic increase in spectral efficiency known as spatial multiplexing gain. Many real-world channels are characterized by a deterministic or line-of-sight component. In such cases, Rician fading conditions hold. The presence of a Rician component tends to reduce the multiplexing gain by reducing the rank of the MIMO channel matrix realizations. Shu-Ming and the group did a comprehensive work [4] when they proposed multiple signature sequences (instead of one) per user in a multicarrier directsequence spread spectrum multiple access system in Rician fading channels. Every user has a distinct set of spreading sequences, with a different spreading sequence for each carrier in each user's set; they further indicated that when these sets of sequences were chosen to be mutually orthogonal complementary sets of sequences, multiple access interference (MAI) was reduced in Rician fading channels. In addition when symbols are packed more closely together results in a higher data rate. In conclusion, a distinct set of deterministic spreading sequences per user had lower MAI and higher data rate than another system with one distinct random spreading sequence per user in Rician fading channels. The authors in [5] dealt with the training-based channel estimation scheme in Rician distributed flat fading multiple-input multiple-output (MIMO) channels. They proposed a new biased shifted scaled least squares (SSLS) technique that was generalized form of the scaled least squares (SLS) approach. This technique was suitable for estimation of both Rayleigh and Rician fading MIMO channels and the performance of the conventional least squares (LS) and SSLS channel estimators was investigated. Moreover, the optimal choice of training sequences was probed using mean square error (MSE) criteria. Analytical and numerical results showed that the proposed SSLS estimator significantly outperformed the LS and SLS techniques. It was also demonstrated that increasing the Rice factor lead to decreasing the MSE of offered technique, while the performance of LS and SLS estimators were independent of this factor. Li-Chun et al in their Capacity fades analysis of MIMO Rician channels in mobile ad hoc networks uses double-ring scattering model with LOS components, authors, suggested a sum-of-sinusoids MIMO mobile-to-mobile channel simulation method, which can characterize the spatial/temporal channel correlation and Rician fading effect. 
Their numerical results showed the ergodicity of our proposed channel model, correctness of the analytical channel correlation, effect of spatial correlation on channel capacity, impact of the number of antennas and scatterers on capacity, capacity distribution, and level crossing. The paper in [6], based on the double-ring scattering model with LOS components the researchers suggested a sum-of-sinusoids MIMO mobile-tomobile channel simulation method, which could characterize the spatial/temporal channel correlation and Rician fading effect. Their numerical results show the ergodicity of their proposed channel model. On the capacity and BER performance of multiuser scheduling over MIMO Nakagami- $m$ fading channels presented by authors in [7] presented a performance analysis for evaluating multiuser and spatial diversities achieved in multiuser MIMO systems employing orthogonal space-time block coding (OSTBC) over Nakagami- $m$ fading channels. Two multiuser scheduling schemes were considered: Absolute SNR-based scheduling (AS) and normalized SNR-based scheduling (NS) schemes for both heterogeneous and homogeneous wireless networks. Analytical expressions were derived for the average channel capacity and average bit error rate (BER) of these systems. The considered scheduling schemes were numerically evaluated and compared in terms of average capacity, average BER and fairness. It was shown that in the heterogeneous case, unlike the AS scheme, the NS scheme can guarantee fairness to the users. It was also shown that in the heterogeneous case, the AS scheme yielded a higher average capacity and a lower average BER compared to the NS scheme and to the homogeneous case. The performance of transmit diversity (TD) assisted amplify-and-forward (AF) relay system with partial relay selection, which experiences mixed Rayleigh and Rician fading channels, was investigated in [8]. In this paper we investigate the effects on variance and multiple interference on the multiple-input multiple-output (MIMO) wireless systems in Uncorrelated Racian interference Channel using the successive decoding approach The analysis was done by splitting the total received power components into direct and diffuse parts before defining the $S I R$ and $S N R$.

The remaining of the paper is organized as follows: Section II presents the MIMO system and the channel models. In Section III the Uncorrelated Racian environment is discussed. Results and Discussions are presented in Section IV with Section V concluding the paper.

\section{MIMO CHANNEL MODEL}

We consider a narrowband block fading channel with $n_{R}$ receiving antennas, $n_{T}$ transmit antennas from an interfering source (possibly representing several different users) characterized by [9]

$$
y=\varsigma \omega+C I \omega I+z .
$$

here, $\boldsymbol{y} \in \mathbb{C}^{n_{R}}$ is the received signal vector $\boldsymbol{\omega} \in \mathbb{C}^{n_{T}}$ is the transmitted complex, Gaussian distributed signal vector with zero mean and covariance $ב=E\left[\boldsymbol{\omega}^{\complement}\right], \boldsymbol{\omega}_{I} \in \mathbb{C}^{n_{I}}$ is the interfering complex. Gaussian distributed signal vector with zero mean and covariance $\beth_{I}=\mathrm{E}\left[\boldsymbol{\omega}_{I} \boldsymbol{\omega}_{I}^{\mathrm{C}}\right]$, and $\boldsymbol{z} \in \mathbb{C}^{n_{R}}$ is additive zero-mean white noise with iid entries $z_{a} \sim \mathcal{N}_{c}(\mathbf{0}, \mathbf{1})$. The channel matrices $\boldsymbol{\zeta} \in \mathbb{C}^{n_{R} \times n_{T}}$ and $\boldsymbol{\zeta}_{I} \in \mathbb{C}^{n_{R} \times n_{I}}$ model separately (or Kronecker) correlated Rician fading. Thus, they can be written as

$$
\left\{\begin{array}{c}
\boldsymbol{C}=\overline{\boldsymbol{C}}+\boldsymbol{p}^{\mathbf{1} / \mathbf{2}} \boldsymbol{\zeta}_{w} \boldsymbol{\Psi}^{1 / 2} \\
\boldsymbol{C}=\overline{\boldsymbol{C}}_{I}+\boldsymbol{p}_{I}^{1 / 2} \boldsymbol{C}_{w, I} \boldsymbol{\Psi}_{I}^{1 / 2}
\end{array}\right.
$$

Where $\overline{\boldsymbol{\zeta}}$ and $\overline{\boldsymbol{\zeta}}_{I}$ represent the mean values of $\boldsymbol{\boldsymbol { C }}$ and $\boldsymbol{\boldsymbol { C }}_{I}$, respectively, and are related to the presence of LOS components, $\left(\boldsymbol{\varsigma}_{w}\right)_{a b},\left(\boldsymbol{\varsigma}_{w, I}\right)_{a b} \sim \mathcal{N}_{c}(\mathbf{0}, \mathbf{1})$, and the positive semidefinite matrices $\boldsymbol{\Psi}\left(\boldsymbol{\Psi}_{I}\right)$ and $\boldsymbol{p}\left(\boldsymbol{p}_{I}\right)$ are the transmit signal (interference) and receive signal (interference) spatial correlation matrices, respectively. The covariance between the different entries of $\boldsymbol{\varsigma}$ and $\boldsymbol{\zeta}_{I}$ satisfies the identities

$$
\left\{\begin{array}{c}
\operatorname{cov}\left((\boldsymbol{C})_{i j}(\boldsymbol{C})_{i^{\prime} j^{\prime}}\right)=(\boldsymbol{p})_{i i^{\prime}}(\boldsymbol{\Psi})_{j j^{\prime}}^{*} \\
\operatorname{cov}\left(\left(\boldsymbol{C}_{\boldsymbol{I}}\right)_{i j}\left(\boldsymbol{C}_{\boldsymbol{I}}\right)_{i^{\prime} j^{\prime}}\right)=\left(\boldsymbol{p}_{\boldsymbol{I}}\right)_{i i^{\prime}}\left(\boldsymbol{\Psi}_{\boldsymbol{I}}\right)_{j j^{\prime}}^{*}
\end{array}\right.
$$

(Extension to multiple interfering transmitters) (3) Can be applied to model multiple interfering transmitter under the assumption of a common receive correlation matrix for each interfering source. Indeed, assume that we have $N_{I}$ interfering users, so that the channel of interfering is $i\left(i=1, \ldots, N_{I}\right)$ of the form

$$
\boldsymbol{\zeta}_{I}^{i}=\overline{\boldsymbol{\zeta}}^{i}+\boldsymbol{p}^{1 / 2} \boldsymbol{W}^{\boldsymbol{i}} \boldsymbol{\Psi}_{\boldsymbol{I}}^{\mathbf{i} / \mathbf{2}} \text { and } \boldsymbol{y}=\boldsymbol{\zeta} \boldsymbol{\omega}+\sum_{i=1}^{N_{I}} \boldsymbol{\zeta}_{I}^{i} \boldsymbol{\omega}_{I}^{i}+\boldsymbol{z}
$$

Then set $\left.\boldsymbol{\omega} \triangleq\left[\boldsymbol{\omega}_{I}{ }^{1 \mathrm{~T}}, \ldots, \boldsymbol{\omega}_{I}{ }^{N_{\mathrm{I}}}\right]^{\mathrm{T}}\right]^{\mathrm{T}}, \quad \boldsymbol{\Psi} \triangleq \bigoplus_{i=1}^{N_{I}} \boldsymbol{\Psi}_{I}^{i}, \quad \boldsymbol{W} \triangleq$ $\left[\boldsymbol{W}^{1}, \ldots, \boldsymbol{W}^{N_{1}}\right]$, and $\overline{\boldsymbol{C}} \triangleq\left[\overline{\boldsymbol{C}}^{1}, \ldots, \overline{\boldsymbol{\boldsymbol { C }}}^{N_{1}}\right]$.

Different receive correlation matrices for the interfering transmitter can be modelled by introducing virtual delays in combination with a wideband channel model, as proposed in [9] [10] and [11].

Following the approach in [12], we define

$$
\left\{\begin{array}{c}
\grave{\boldsymbol{C}} \triangleq \overline{\boldsymbol{C}} \beth^{1 / 2} \grave{\boldsymbol{\Psi}}=\boldsymbol{\Psi}^{1 / 2} \beth \boldsymbol{\Psi}^{1 / 2} \\
\grave{\boldsymbol{\zeta}} \triangleq \grave{\boldsymbol{\zeta}}_{I} \beth_{I}{ }^{1 / 2} \grave{\boldsymbol{\Psi}}_{I}=\boldsymbol{\Psi}_{I}{ }^{1 / 2} \beth_{I} \boldsymbol{\Psi}_{I}{ }^{1 / 2}
\end{array}\right.
$$

Then, the transmitted signal and interference covariance matrices are implicitly accounted for into $\grave{\boldsymbol{\zeta}}, \grave{\boldsymbol{\zeta}}_{I}, \grave{\boldsymbol{\Psi}}_{\text {and }} \grave{\boldsymbol{\Psi}}_{I}$. According to these definitions, the total received power is given by

$$
\begin{aligned}
& \mathrm{E}\left[\|\boldsymbol{y}\|^{2}\right]=\|\dot{\boldsymbol{\zeta}}\|^{2}+\operatorname{tr}(\boldsymbol{p}) \operatorname{tr}(\grave{\boldsymbol{\Psi}}) \\
& +\left\|\dot{\boldsymbol{\aleph}}_{I}\right\|^{2}+\operatorname{tr}\left(\boldsymbol{p}_{\boldsymbol{I}}\right) \operatorname{tr}\left(\grave{\boldsymbol{\Psi}}_{I}\right)+n_{R}
\end{aligned}
$$

Splitting the total received power components into direct and diffuse parts we obtain Rician factors

$$
K=\frac{\|\dot{\boldsymbol{\zeta}}\|^{2}}{\operatorname{tr}(\boldsymbol{p}) \operatorname{tr}(\grave{\boldsymbol{\Psi}})} K_{I}=\frac{\left\|\dot{\boldsymbol{\varphi}}_{I}\right\|^{2}}{\operatorname{tr}\left(\boldsymbol{p}_{I}\right) \operatorname{tr}\left(\grave{\boldsymbol{\Psi}}_{I}\right)}
$$

Then, we can define the signal-to-noise and interference-to-noise power ratios as

$$
\left\{\begin{array}{l}
S N R=\frac{(K+1) \operatorname{tr}(\boldsymbol{p}) \operatorname{tr}(\grave{\boldsymbol{\Psi}})}{n_{R}} \\
I N R=\frac{\left(K_{I}+1\right) \operatorname{tr}(\boldsymbol{p}) \operatorname{tr}\left(\grave{\boldsymbol{\Psi}}_{I}\right)}{n_{R}}
\end{array}\right.
$$

with signal-to-interference power ratio. It can be noticed that the previous definitions of the Rician factors and of the SNRs in (3) and (4) depend on the full transmit covariance matrices בand 
$\beth_{I}$ and not only on their traces, unless they are proportional to the identity matrix. This may be an issue when the channel capacity is evaluated against the SNR since Iis not proportional to the identity matrix if it is optimized to achieve the ergodic capacity $[9,12]$

In what follows we further assume that:

1) The spatial correlation matrices are normalized by

$$
\begin{gathered}
\operatorname{tr}(\boldsymbol{p})=\operatorname{tr}\left(\boldsymbol{p}_{I}\right)=n_{R} \\
\operatorname{tr}(\boldsymbol{\Psi})=n_{T} \\
\operatorname{tr}\left(\boldsymbol{\Psi}_{I}\right)=n_{I} .
\end{gathered}
$$

2) The matrices defined in (2) are normalized according to the identities

$$
\left\{\begin{array}{c}
\|\dot{\boldsymbol{C}}\|^{2}=\frac{n_{R} K S N R}{K+1} \operatorname{tr}(\grave{\boldsymbol{\Psi}})=\frac{S N R}{K+1} \\
\left\|\dot{\boldsymbol{C}}_{I}\right\|^{2}=\frac{n_{R} K_{I} I N R}{K_{I}+1} \operatorname{tr}\left(\grave{\boldsymbol{\Psi}}_{I}\right)=\frac{I N R}{K_{I}+1}
\end{array}\right.
$$

\section{UNCORRELATED RICIAN FADING}

If the MIMO channel is subject to URiF, then, as stated in Section III, the $n_{R} \times n_{R}$ random matrix $\lambda \lambda^{\mathcal{H}}$ follows a Wisharttype distribution. By defining the $m \times m$ random matrices Sand $\boldsymbol{\Omega}$ such that

$\mathbf{S}:=\left\{\begin{array}{l}\lambda \lambda^{\mathcal{H}}, \text { if } n_{T} \geq n_{R} \\ \lambda^{\mathcal{H}} \lambda, \text { if } n_{T}<n_{R}\end{array}:=\left\{\begin{array}{l}\mathrm{NMM}^{\mathcal{H}}, \text { if } n_{T} \geq n_{R} \\ \mathrm{NM}^{\mathcal{H}} \mathrm{M}, \text { if } n_{T}<n_{R}\end{array}\right.\right.$

(6)

it is easily seen that the distribution of the nonzero eigenvalues of $\lambda \lambda^{\mathcal{H}}$ is determined by the eigenvalue distribution of the complex noncentral Wishart matrix $\mathbf{S} \sim \mathcal{C W}\left(n, \frac{1}{K+1} \boldsymbol{I}_{m}, \boldsymbol{\phi}\right)$. Assuming that \$has $t>0$ distinct eigenvalues $\omega_{m-t+1}, \ldots, \omega_{m}$, denoted as the column-vector $\omega_{t}=\left[\omega_{m-t+1}, \ldots, \omega_{m}\right]^{\mathrm{T}}$, then, following the approach in [9], [13] and [14], it can be shown that the PDF of a single unordered eigenmode SNR is given by

$$
\begin{gathered}
p_{\gamma 1}(\gamma)= \\
\frac{\aleph_{m, n}^{\omega}}{m} \sum_{i=1}^{m} \mu_{\aleph}[\mathrm{O}+\tilde{\omega}] \\
\mathrm{O}=\sum_{j}^{1} \sigma_{i, j}^{(t)}\left(\mu_{\aleph \gamma}\right)^{d+i+j-2} e^{-\mu_{\aleph \gamma}}
\end{gathered}
$$

$$
\tilde{\omega}=\sum_{j}^{2} \sigma_{i, j}^{(t)}\left(\mu_{\aleph \gamma}\right)^{d+i-1} e^{-\mu_{\aleph \gamma}} \bar{F}_{1}\left(d+1 ; \mu_{\aleph \gamma \omega_{j}}\right)
$$

where,

$$
\mu_{\aleph}:=\frac{(\aleph+1)}{\bar{\gamma}},\left(\aleph_{m, n}^{\omega_{t}}\right)^{-1}:=\eta \dddot{\alpha} \hat{\alpha} \hat{\omega}
$$

$$
\dddot{\eta}=V\left(\boldsymbol{\omega}_{t}\right) e^{\sum_{j=m-t+1}^{m} \boldsymbol{\omega}_{j}}
$$

$$
\grave{\alpha}=\prod_{j=1}^{m-t} \Gamma(d+j) \Gamma(j)
$$

$$
\hat{\varphi}=\prod_{j=m-t+1}^{m} \boldsymbol{\omega}_{j}^{m-t}
$$

With $V(\boldsymbol{\omega} t)$ denoting the Van Der Monde determinant of order $t$ associated with $\boldsymbol{\omega} t$ and $\Gamma($.$) the standard Gamma function [15]$

$$
{ }_{0} \bar{F}_{1}(b ; 3):=\sum_{k=0}^{+\infty} \frac{3^{\aleph}}{\Gamma(b+\kappa) \aleph !}
$$

is a regularized confluent hypergeometric limit function, $\sigma(t)_{i j}^{(t)}:=(-1)^{i+j} \operatorname{det}\left[\boldsymbol{\sigma}_{i j}\left(\boldsymbol{\omega}_{t}\right)\right]_{m-1}$, with $\sigma_{i j}(\boldsymbol{\omega} t)$ denoting the $(i, j)^{\text {th }}$ minor of $\quad \sigma_{i j}(\boldsymbol{\omega} t):=$ $\left(\begin{array}{c}\Gamma(d+i+j-1) \Gamma(d+i)_{1} \bar{F}_{1}(d+i ; d+1 ; \omega j) \\ j \leq m-t j>m-t\end{array}\right)_{m}$ obtained by removing the $i^{t h}$ row and $j^{\text {th }}$ column thereof and ${ }_{1} \bar{F}_{1}(a ; b ; 3):=\sum_{\aleph=0}^{+\infty} \frac{\Gamma(a+k) 3_{3}{ }^{\aleph}}{\Gamma(a) \Gamma(b+k) \aleph !}, d:=n-m$ and we have introduced the shorthand notations $\sum_{j}^{1}:=\sum_{j=1}^{m-t}$ and $\sum_{j}^{2}:=\sum_{j=m-t+1}^{m}$ for convenience.

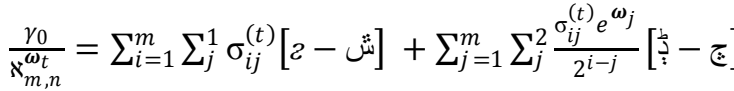

(14)

$2=\Gamma\left(d+i+j-1, \mu_{\aleph} \gamma_{0}\right)$

离 $=\mu_{\aleph} \gamma_{0} \Gamma\left(d+i+j-2, \mu_{\aleph} \gamma_{0}\right)$

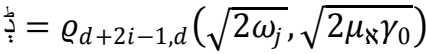

ج $=2 \mu_{\aleph} \gamma_{0} \varrho_{d+2 i-3, d}\left(\sqrt{2 \omega_{j}}, \sqrt{2 \mu_{\aleph} \gamma_{0}}\right)$

$\sigma_{e m-o p r a}^{m, n}=m \frac{E_{1}\left(\mu_{\aleph} \gamma_{0}\right)}{\operatorname{In}(2)}+\frac{\aleph_{m, n}^{\omega_{t}}}{\operatorname{In}(2)} \sum_{i=1}^{m}[\not K+\hat{\mathcal{j}}]$

$L K=\sum_{j}^{1} \sigma_{i j}^{(t)} \Gamma(d+i+j-1) \sum_{l=1}^{d+i+j-2} \frac{\Gamma\left(l, \mu_{\aleph} \gamma_{0}\right)}{\Gamma(l+1)}$

$\widehat{C}=\sum_{j}^{2} \sigma_{i j}^{(t)} \sum_{k=0}^{+\infty} \frac{\Gamma(d+i+k) \omega_{j}^{k}}{\Gamma(k+1) \Gamma(d+1+k)} \sum_{l=1}^{d+i+k-1} \frac{\Gamma\left(l, \mu_{\aleph} \gamma_{0}\right)}{\Gamma(l+1)}$ 
Substituting (7) into $\int_{\gamma_{0}}^{+\infty}\left(\frac{1}{\gamma_{0}}-\frac{1}{\gamma}\right) p_{\gamma 1}(\gamma) d \gamma=\frac{1}{m}$, it is seen that the optimal cutoff value has to satisfy $(14)$ where $\Gamma(a, 3):=$ $\int_{3}^{+\infty} t^{a-1} e^{-t} d t$ denotes the upper incomplete Gamma function and $\varrho_{p, q}(a, b)$ is the normalized Nuttall $Q$ function, defined as $\varrho_{p, q}(a, b):=Q_{p, q}(a, b) / a^{q}$, where $\varrho_{p, q}(a, b)$ stands for the standard Nuttall $Q$-function [16]

$$
\begin{gathered}
Q_{p, q}(a, b)=\int_{b}^{\infty} 3^{p} \exp \left(-\frac{3^{2}+a^{2}}{2}\right) I_{q}\left(a_{3}\right) d 3_{3} \\
0<a, b<\infty, p, q=0,1,2, \ldots
\end{gathered}
$$

with $I_{q}($.$) being a modified Bessel function of the first kind.$ Existence and uniqueness of a $\gamma_{0}$ satisfying (8) can be easilyproved following a similar approach to the one used by theauthors in [32]. In order to evaluate the capacity of the emopra transmission policy, we substitute (7) into $\sigma_{\text {em-opra }}^{m, n}=$ $m \int_{\gamma_{0}}^{+\infty} \log _{2}\left(\frac{\gamma}{\gamma_{0}}\right) p_{\gamma 1}(\gamma) d \gamma$, then replace ${ }_{0} \bar{F}_{1}(. ;$. . ) with its infinite series representation, and exchange the integral and summation orders which yields (9) at the top of next page after some further manipulation, where $E 1(\mu):=\int_{1}^{+\infty} \frac{e^{-\mu t}}{t} d t$ denotes the exponential integral function of the first order. The outage probability hinges on the cumulative distribution function (CDF) of the largest eigenvalue of $\lambda \lambda^{\mathcal{H}}$ [9],[18].

Accordingly, it can be shown that the outage probability induced by the em-opra policy can be expressed as functions of $\gamma_{0}$ as

$P_{\text {out }}^{m, n}=\operatorname{Prob}\left(\gamma_{\max } \leq \gamma_{0}\right)=P\left(\mu_{K} \gamma_{0}\right)$

(23)

where $P(3):=K_{m, n}^{\boldsymbol{\omega}_{\boldsymbol{t}}} \operatorname{det}[\boldsymbol{D}(3)]_{m}$, and the elements of the $m \times m$ matrix $\boldsymbol{D}(3)$ for $i, j=1, \ldots, m$ are given by

$$
\begin{aligned}
& \{\mathbf{D}(3)\}_{i j} \\
& =\left\{\begin{array}{r}
\gamma(d+i+j-1, x), i f j=1, \ldots, m-t \\
\Gamma(d+i){ }_{1} \bar{F}_{1}\left(d+i ; d+1 ; \omega_{j}\right) \\
-\frac{e^{\omega j}}{2^{i-1}} \varrho_{d+2 i-1, d}\left(\sqrt{2 \omega_{j}}, \sqrt{23}\right), \quad \text { otherwise. }
\end{array}\right.
\end{aligned}
$$

where, $\quad \gamma(a, 3):=\Gamma(a)-\Gamma(a, 3)$

\section{RESULTS AND DISCUSSION}

The analysis was done by splitting the total received power components into direct and diffuse parts before defining the $S I R$ and $S N R$. The extension of MIMO system with $n_{R}$ receive antennas and $n_{T}$ transmit antennas to multiple interfering transmitter under the assumption of a common receive correlation matrix indicates that the Racian factor and SNR depend on the full transmit covariance matrices ${ }$ and $\beth_{I}$ and not only their traces, unless they are proportional to identity matrix.

\section{CONCLUSION}

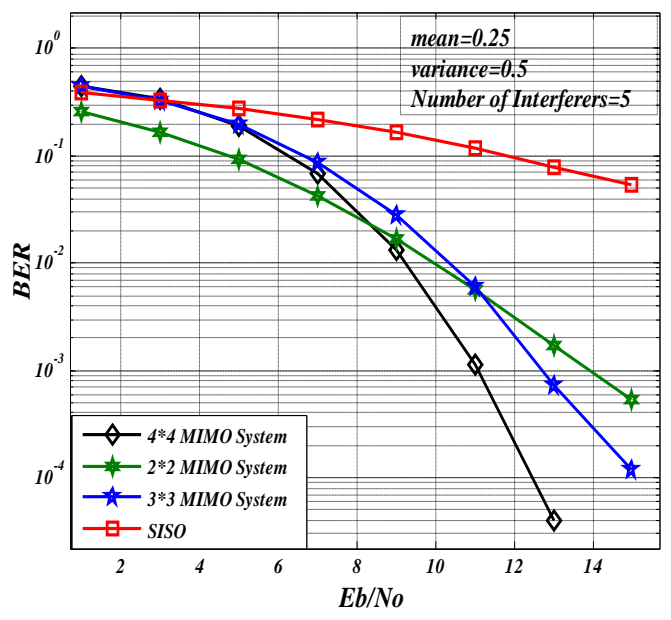

Fig 1: Performance of MIMO Systems with 5 Interferes

The problem of evaluating the BER of uncorrelated Racian channel for MIMO system affected multiple interference has been analyzed instead of Evaluation of BER in correlated Racian environment for MIMO systems and Ergodic Capacity of Correlated Racian Fading MIMO Channels with Interference review in the previous literature work with multiple interference. The simulation results using the Monte Carlo approach indicates that the variation of the mean improves the MIMO performance with the number of interferers having less effect on the BER performance of the System. This complements the earlier results made by Erwin et all when dealing with Asymptotic Statistics of the Mutual Information for correlated Racian Fading MIMO channels with interference[10]. Also, the research seems to be important for large number of Antennas as the performance of the $4 * 4$ MIMO system seems less affected by the variance and the number of interfere

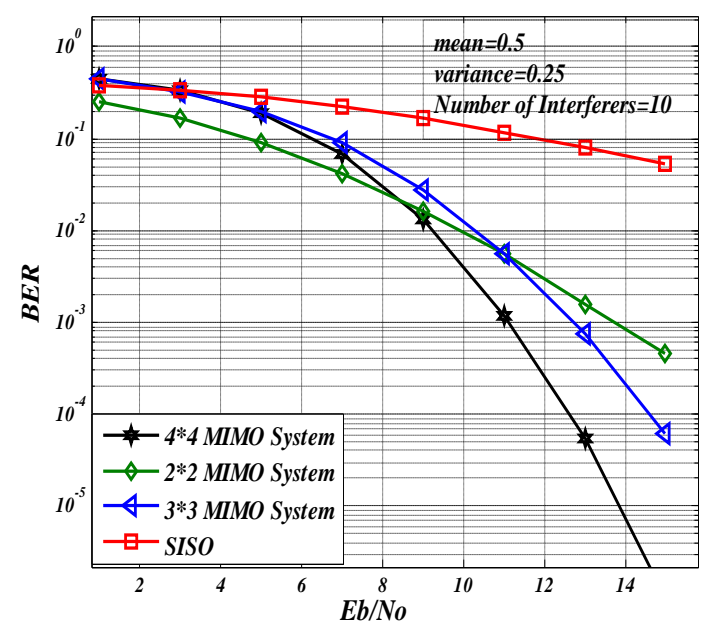

Fig 2: Performance of MIMO Systems with 10 Interferes 


\section{REFERENCES}

[1] Volker K., "Wireless Communication Over MIMO Channels", Application to CDMA and Multiple Antenna Systems, John Wiley and Sons, Ltd., South Gate, Chichester, England, pp. 1,2, 2006

[2] Telstar I.E., "Capacity of Multi-antenna Gaussian Channels", Europe. Trans. Telecommunication., vol. 10, no. 6, pp. 586-595, Nov. /Dec. 1999.

[3] Foschini G. J., Gans M. J., "On limits of Wireless Communications in a Fading Environment when using Multiple Antennas," Wireless Personal Communications. vol. 6, no. 3, pp. 311-335, Mar. 1998.

[4] Shu-Ming T., Chuang-Chun C., "A multicarrier DS/SSMA system with reduced multiple access interference and higher data rate in Rician fading channels", Signal Processing, Vol.81, Issue 9, September 2001, pp. 1889-1897

[5] Hamid N., Shahriar S. M., "A novel shifted type of SLS estimator for estimation of Rician flat fading MIMO channels", Signal Processing, Vol. 90, Issue 6, June 2010, pp.1886-1893

[6] Li-Chun W., Wei-Cheng L., Yun-Huai C.," Capacity fades analysis of MIMO Rician channels in mobile ad hoc networks", Performance Evaluation, Vol. 66, Issue 12, December2009,pp.742-753

[7] Mohammad T., David H., Wessam A., “ On the capacity and BER performance of multiuser scheduling over MIMO Nakagami-m fading channels", Signal Processing,Vol.91, Issue11, November 2011, pp. 24872496

[8] Wei X., Jian-hua Z., Ping Z.,"Performance of transmit diversity assisted amplify-and-forward relay system with partial relay selection in mixed Rayleigh and Rician fading channels", The Journal of China Universities of Posts and Telecommunications, Vol.18, Issue 5, October 2011, pp. 37-41,46

[9] Erwin, R.,and Giorgio, T., "Asymptotic Statistics of the Mutual Information for Spatially Correlated Racian Fading MIMO Channel with Interference" IEEE Transaction on Information Theory, Vol. 75, No. 7, April 2010

[10] Riegler E., Taricco G., "Asymptotic ergodic capacity region and rate optimization of a multiple access OFDM MIMO channel with separately correlated Rician fading," in Proc. IEEE GLOBECOM 2008, New Orleans, LA, Nov. 30-Dec. 42008

[11] Riegler E., Taricco G., "Asymptotic ergodic capacity of wideband MIMO channels with separately correlated Rician fading," in Proc. IEEE GLOBECOM 2008, New Orleans, LA, Nov. 30-Dec. 42008

[12] Taricco G., "Asymptotic mutual information statistics of separately correlated Rician fading MIMO channels," IEEE Trans. Inf. Theory, vol.54, no. 8, pp. 3490-3504, Aug. 2008
[13] Maaref A., Aissa S., "On the capacity statistics of MIMO Ricean and Rayleigh fading channels," in Proc. IEEE Int. Conf. on Communications (ICC'06), Istanbul, Turkey, June 2006, in press.

[14] Alfano G., Lozano A., Tulino A. M., Verd'u S., "Mutual information and eigenvalue distribution of MIMO Ricean channels," in Proc. IEEEInt. Sump. on Inform. Theory \& Applications (ISITA'04), Parma, Italy, Oct. 2004, pp. 10401045.

[15] Gradshteyn I. S., Ryzhik I. M., "Table of Integrals, Series, and Products," 6th ed. San Diego, California: Academic Press, 2000

[16] Nuttall A. H., "Some integrals involving the Q function," Naval Underwater Systems Centre, New London Lab., New London, CT, 4297, 1972.

[17] Maaref A., A1ssa S., "On the achievable spectral efficiency of adaptive transmission with transmit-beam forming," in Proc. IEEE Int.Conf. on Commun. (ICC'05), Seoul, Korea, May 2005, pp. 2287-2291.

[18] JayaweeraS. K., Poor H. V., "Capacity of multiple-antenna systemswith both receiver and transmitter channel state information," IEEETrans. Inform. Theory, vol. 49, no. 10, pp. 2697-2709, Oct. 2003.

[19] Giorgio, T. and Erwin, R., "On the Capacity of Correlated Racian Fading Channel with Interference" IEEE Transaction on Information Theory, Vol. 75, No. 7, July 2011

[20] Yifei Z., Ming Z., Limin X., Jing W. T., "Analytical Expression for the MIMO Channel Capacity", Science \& Technology, Vol.11, Issue 3, June 2006, pp. 271-277

[21] Yong-hua L., Tie-jun L., Xiao-fei W., Lin Z.," On the bounds of frequency-selective channel capacity with doubly correlated geometrical MIMO channel model", The Journal of China Universities of Posts and Telecommunications, Vol. 17, Issue 2, April 2010, pp.8-13

[22] E. Affum Ampoma, Dr. T.R. Rao, Dr. V. A. Labay "Capacity and performance analysis of a MIMO based MB-OFDM Ultra wide band Communication System", $2^{\text {nd }}$ International Conference on Adaptive Science \& Technology pages 432-439, Dec. 2009 Accra

[23] E. Affum Ampoma, Dr. Reynolds Okai, Dr. Stanley Moffat "Uplink Performance Analysis in Multiple MIMO Rayleigh Interference Channel for WCDMA", International Conference on Wireless Networks , WORLDCOMP'11 (ICWN'11), Conference Proceedings pages 16-21, (18th -21st) July 2011, Las Vegas, Nevada, USA 